\title{
Clinical characteristics of OGTT-derived hepatic- and muscle insulin resistance in healthy young men
}

\author{
Maeng Kyu Kim* \\ Department of Physical Education, Kyungpook University, Daegu, Korea
}

(Received: 2014/07/31, Revised: 2014/11/26, Published online: 2014/12/10)

Maeng Kyu Kim. Clinical characteristics of OGTT-derived hepatic- and muscle insulin resistance in healthy young men. JENB., Vol. 18, No. 4, pp.385-392, 2014 [Purpose] Insulin inhibits glucose release in the liver but increases glucose absorption in muscles. When insulin cannot properly control glucose, it negatively affects glucose metabolism and, furthermore, contributes to the onset of metabolic syndrome and chronic disease. Therefore, this study's goal is to understand the clinical characteristics of hepatic insulin resistance and muscle insulin sensitivity in healthy young men. [Methods] Twenty-eight healthy young men (age 23.3 \pm 0.5 ; mean $\pm \mathrm{SE}$ ) participated in this study. Liver function and blood lipids were measured by blood sampling from brachial vein after participants fasted the previous day. Hepatic insulin resistance and muscle insulin sensitivity were evaluated using two-hour OGTT along with surrogate index related to insulin sensitivity. The $\mathrm{VO}_{2 \max }$ was evaluated using cycle ergometer. Systemic insulin sensitivity was evaluated using two-hour euglycemic hyperinsulinemic clamp method. [Results] Hepatic insulin resistance showed a significant correlation with body fat $(\mathrm{r}=0.609, p<0.05)$. Also, hepatic insulin resistance showed a significant correlation with GOT $(r=0.467)$, GPT $(r=0.434)$, and $\gamma-G T P(r=0.375)$, reflecting liver functions, as well as showing a significant correlation with hs-CRP $(\mathrm{r}=0.492, p<0.05)$. On the other hand, muscle insulin sensitivity had no correlation with neither body fat nor liver function index $(p>0.05)$, and among surrogate indexes, it showed a significant correlation with Avignon $(r=-0.493)$ and Matsuda index $(\mathrm{r}=-0.577)$. Glucose infusion rate, using the clamp method, showed a significant correlation with muscle insulin sensitivity $(\mathrm{r}=0.448, p<0.05)$. The $\mathrm{VO}_{2 \max }$ had a significant correlation with hepatic insulin resistance $(\mathrm{r}=-0.435, p<0.05)$ and muscle insulin sensitivity $(\mathrm{r}=0.474, p<0.05)$, respectively. [Conclusion] For young men in their 20's, the OGTT-based hepatic insulin sensitivity was an indicator of hepatic function and body fat but muscle insulin sensitivity was related to peripheral insulin sensitivity. Also, for young men, higher $\mathrm{VO}_{2 \max }$ indicated lower hepatic insulin resistance and higher muscle insulin sensitivity. [Key words] hepatic insulin resistance, muscle insulin sensitivity, $\mathrm{VO}_{2 \max }$, surrogate index

\section{INTRODUCTION}

Blood sugar in the body (glucose) is the main fuel of the body and is used in all the tissues. Especially, glucose is the only energy source for the brain and blood glucose level remained consistently in a narrow range $(70-90 \mathrm{mg} / \mathrm{dl})$ [1]. In such blood glucose homeostasis, blood insulin plays an optimal role. For glucose kinetics, insulin in the body generally inhibits hepatic glucose secretion and increases glucose absorption in the muscle. In the quantitative aspect, insufficient insulin secretion of pancreatic beta cells increases blood glucose and causes type 1 diabetes [2] while insulin resistance, which is increased in peripheral organs, such as the liver and muscle, not only plays a major role in the onset of type 2 diabetes but also contributes to the onset of hypertension and hyperlipidemia-related metabolic syndromes and chronic disease [3]. Therefore, insulin not only controls blood glucose but also plays a very important role in the body for homeostasis.

Insulin resistance is a state where insulin hormone-mediated glucose absorption and the response to insulin metabolism, which inhibits hepatic gluconeogenesis, are reduced, and, in general, it describes the state of reduced insulin sensitivity $[4,5]$. The effects and functions of the decrease of insulin on tissues of the body, are described below. First, insulin inhibits hepatic glucose secretion, but, if such effect is

\footnotetext{
* Corresponding author: Maeng Kyu Kim, Tel. 82-53-950-5937, Fax: 053-955-4235, Email: kimmk@knu.ac.kr

(c)2014 The Korean Society for Exercise Nutrition
} 
insufficient, hepatic insulin resistance caused by decreased insulin activity will cause hyperglycemia [6]. Second, it increases glucose absorption in muscle cells. Although insulin plays an important role in the absorption (elimination) of glucose, decreased insulin action on muscle, i.e. muscle insulin resistance, is a characteristic of metabolic syndrome and diabetes patients [7]. Therefore, the study of insulin action on the liver and muscle tissues is very important in terms of understanding the mechanism of pathophysiology.

There are various methods to evaluate insulin resistance. The 'so called' Golden standard method, euglycemic hyperinsulinemic clamp method was developed by DeFronzo for the first time and it inhibited hepatic glucose secretion using high-insulin injection and thus evaluates peripheral insulin sensitivity $[4,8]$. Also, there was a minimal model method which was much less invasive (injecting glucose and insulin bolus into venous blood) than the clamp method $[9,10]$. Although the clamp method and the minimal model are the best methods to evaluate insulin resistance, they take a lot of time, money, and labor and are not easy for a large-scale evaluation in vivo on the human body due to the use of isotopes and complicated test methods to measure insulin resistance of each tissue group.

On the contrary, surrogate index for insulin sensitivity has been widely used. In general, the surrogate indexes using fasting blood glucose and insulin value, such as HOMA (The homeostatic model assessment) and QUICKI (A quantitative insulin sensitivity check index), are used but, for more comprehensive assessment of glucose kinetics in the body, Oral Glucose Tolerance Test (OGTT) has been used. Interestingly, Abdul-Ghani, et al. reported an equation that can evaluate hepatic insulin resistance and muscle insulin sensitivity at the same time using two-hour OGTT data values [11]. This method assessed insulin resistance and sensitivity on each tissue safely and easily, and it was relatively cheap and more efficient than the clamp method and the minimal model method. From numerous study results, $\mathrm{VO}_{2 \max }$ (maximal oxygen consumption) reflecting cardiovascular fitness was related to insulin sensitivity $[12,13]$. Low $\mathrm{VO}_{2 \max }$ was used as an initial clinical indicator for the people with insulin resistance syndrome or type 2 diabetes, and, furthermore, it iwa acknowledged that the decrease of $\mathrm{VO}_{2 \max }$ was an important risk factor related to various diseases' progression [14].

One previous study reported a positive correlation between $\mathrm{VO}_{2 \max }$ and systemic insulin sensitivity caused by the clamp method (glucose removal rate by insulin stimulation) [15], but, still, there were not many reports on the correlation between insulin sensitivity and $\mathrm{VO}_{2 \max }$ on each tissue. As previously stated, the clamp method without using isotopes cannot directly evaluate hepatic and muscle insulin resistance. Therefore, this study was designed to confirm the correlation between OGTT-based hepatic and muscle insulin resistance along with the euglycemic hyperinsulinemic clamp method, which evaluates systemic insulin resistance, and to study the correlation among each tissue insulin resistance, blood lipids, and existing surrogate indexes. Also, an additional goal of this study was to reveal the correlation between tissue insulin resistance and $\mathrm{VO}_{2 \max }$.

\section{METHODS}

\section{Study subjects and data collection method}

The subjects of this study were the people who lived in the city $\mathrm{J}$, and were recruited from post- and phone-counseling from Jan. 2010 to Mar. 2010. For conducting exercise, the people with orthopedic problems, heart disease, or metabolic disease were excluded from this study. A total of 28 healthy men were recruited (Age 23.3 \pm 0.5 years; BMI $22.8 \pm 0.6$ $\mathrm{kg} / \mathrm{m}^{2}$; Percentage of body fat $17.7 \pm 1.2 \%$ ). The subjects were men who had regular exercise (at least 30 minutes per day and once per week) before participating in this study.

\section{Physical assessment}

Physical assessment was done after 10 hours of fasting. Height was measured with a digital equipment handling up to $0.1 \mathrm{~cm}$ unit and weight was measured with a scale handling up to $0.01 \mathrm{~kg}$ unit while wearing underwear without shoes or socks (TBF-215; Tanita, Tokyo, Japan). BMI was obtained by dividing weight $(\mathrm{kg})$ by height squared $\left(\mathrm{m}^{2}\right)$. Waist circumference was measured twice in a row from the navel area of the subjects and the average value was used. Body fat was measured with a bioelectrical impedance method (Inbody-720; Biospace, Korea).

\section{Maximum aerobic capacity}

Cycle ergometer (818E; Monark, Vansbro, Sweden) was used to assess $\mathrm{VO}_{2 \max }$ of the subjects. To be more specific, a warm-up was done for 2 minutes with $0 \mathrm{~W}$ and exercise was started with $15 \mathrm{~W}$. Loading was done per every 1 minute by $15 \mathrm{~W}$ and the intensity was gradually increased until the subjects could not do anymore. Breathing and breathing gas were assessed using automated gas exchange system (Meta Max, Leipzig, Germany). Exercise stress test was discon- 
tinued in the following situations; 1) rating of perceived exertion (RPE) was 18 or higher, 2) the heart rate during exercise reached $90 \%$ or more of the maximum heart rate calculated based on the age or had extreme tiredness that prevented pedaling, and 3) serious arrhythmia or excessively abnormal ST segment. (ST segment depression of horizontal or downward direction of more than $1 \mathrm{~mm}$ ) [16].

\section{Euglycemic hyperinsulinemic clamp}

After a minimum of 10 to 12 hours of fasting from the previous day, the subjects had a rest of 20-30 minutes in vivo and then intravenous catheters were attached to both arms. One arm was used for injecting glucose and insulin and the other arm was used for blood glucose sampling. Artificial pancreas (STG22; Nikkiso, Shizuoka, Japan) was used for starting euglycemic hyperinsulinemic clamp for two-hours. As with previous studies, the target blood glucose was set at 95 $\mathrm{mg} / \mathrm{dl}$ and insulin was injected with the speed and amount of $100 \mathrm{mU} / \mathrm{m}^{2} / \mathrm{min}$. Between 105 and 120 minutes of the clamp, glucose was automatically injected to maintain the steady-state of the glucose level. Peripheral insulin level was $200 \mu \mathrm{U} / \mathrm{mL}$, which was known to be enough to suppress endogenous gluconeogenesis, and, thus, the glucose infusion rate with the steady-state concentration level was used as an indicator of systemic and peripheral insulin sensitivity [17].

\section{Oral Glucose Tolerance Test (OGTT)}

OGTT was conducted on a different day than the day conducting the clamp method. It was attached by injecting a small polyethylene catheter into the median brachial vein after a minimum of 10 hours of fasting. Then, $75 \mathrm{~g}$ of glucose (TRELAN-G75, Ajinomoto) was taken over 5 minutes and venous blood collecting was done with the catheter every 30 , 60, 90 and 120 minutes after the intake and, then, blood glucose and insulin analysis was immediately done by injecting the blood into a tube containing EDTA $(1 \mathrm{mg} / \mathrm{mL})$.

\section{Blood analysis}

Serum lipids (TC, total cholesterol; HDL-C, high-density lipoprotein cholesterol; FFA, free fat acid; TG, triglyceride) were assessed with an enzymatic assay method, and liver function tests (GOT, GPT, yGTP) were done using ultraviolet (UV) method. Plasma insulin was measured using antibody ELISA (Wako Pure Chemicals. Ltd, Japan). High-sensitivity C-reactive protein (hs-CRP) was measured using Latexturbidimetric immuno-assay.

\section{Hepatic and muscle insulin resistance and sensitivity}

Recently, it became possible to assess tissue insulin sensitivity using OGTT. The method, which was from glucose and insulin value with two-hour OGTT was developed by Abdul-Ghani, et al. [11]. To be more specific, hepatic insulin resistance was calculated with the multiplication of glucose from 0-30 minutes and the surface of insulin (area under the curve; AUC). Muscle insulin sensitivity was calculated by dividing the slope of each natural logarithm of blood glucose on the least-squares regression line by mean insulin value.

\section{Glucose metabolism index (surrogate index)}

OGIS (oral glucose insulin sensitivity), a method developed by Mari, et al., assessed insulin sensitivity using two-hour OGTT [18]. HOMA-IR was the value obtained by the multiplying the fasting insulin $(\mu \mathrm{U} / \mathrm{ml})$ and blood glucose (mg/dl) and then dividing this value by 405 [19]. QUICKI was a surrogate index expressing insulin resistance and calculated by the formula of $1 /[\log ($ insulin, $\mu \mathrm{U} / \mathrm{mL})+\log$ (glucose, mg/dL)] [20]. The HOMA-\%S (insulin sensitivity) and HOMA-\%B (beta cell function) were calculated using Oxford HOMA automatic calculator (www.dtu.ox.ac.uk/ index.php?maindoc $=$ /homa/index.php) [21]. Matsuda index expressing insulin sensitivity was calculated by the value of blood glucose and insulin from OGTT [22]. To be more specific, the formula was 10,000/sqrt (blood glucose $\times$ insulin $\times$ $\mathrm{Gm} \times \mathrm{Im})$ and $\mathrm{Gm}$ was calculated using mean blood sugar level at 0,30 ,and 120 minutes and Im was calculated using mean insulin level at 0, 30, and 120 minutes. Insulin sensitivity index by Avignon, et al. was measured with the same method from the previous report [23]. The FFA-based (free fatty acids) insulin resistance was calculated by the multiplication of fasting plasma FFA (mmol/L) and insulin level (pmol/L) [24].

\section{Data analysis}

The descriptive statistics values of all the data in this study was calculated using SPSS program version 20 (Chicago, USA). Pearson's correlation coefficient (r) was analyzed to evaluate the correlation among measured variables. All the statistical significance levels was set at $a=.05$.

\section{RESULTS}

\section{General characteristics of the subjects}

The physical characteristic of this study was young men 
Table 1. Anthropometric and blood variables in the subjects

\begin{tabular}{lc}
\hline \multicolumn{1}{c}{ Parameter } & Mean \pm S.E \\
\hline Age (years) & $23.29 \pm 0.53$ \\
Body Weight $(\mathrm{kg})$ & $68.09 \pm 2.03$ \\
Body mass index $\left(\mathrm{kg} / \mathrm{m}^{2}\right)$ & $22.79 \pm 0.62$ \\
Waist $(\mathrm{cm})$ & $76.50 \pm 1.66$ \\
Body fat $(\%)$ & $17.69 \pm 1.18$ \\
Fasting glucose (mg/dL) & $86.14 \pm 0.93$ \\
Fasting insulin (gU/mL) & $4.05 \pm 0.38$ \\
Free fatty acids (mEq/L) & $0.44 \pm 0.02$ \\
TC (mg/dL) & $172.07 \pm 5.95$ \\
TG (mg/dL) & $72.61 \pm 5.33$ \\
HDL-C (mg/dL) & $53.21 \pm 2.29$ \\
LDL-C (mg/dL) & $104.34 \pm 4.91$ \\
AST (IU/L) & $19.32 \pm 1.65$ \\
ALT (IU/L) & $21.43 \pm 4.54$ \\
V-GTP (IU/dL) & $24.46 \pm 3.00$ \\
Glycated hemoglobin (\%) & $4.71 \pm 0.03$ \\
\hline
\end{tabular}

Abbreviations: TC, total cholesterol; HDL-C, high-density lipoprotein cholesterol; TG, triglyceride; LDL-C, low-density lipoprotein cholesterol; AST, alanine aminotransferase; ALT, aspartate aminotransferase; $\gamma$-GTP, $\gamma$-glutamyl transpeptidase.

who did regular exercise training as shown in Table 1. Their mean age was 23.3 and mean BMI was $22.8 \mathrm{~kg} / \mathrm{m}^{2}$, and they were recruited among people who were not obese.

The correlation among tissue insulin resistance, body composition, blood lipids, and liver function index

Hepatic insulin resistance showed a significant positive correlation with body fat ( $\%$ fat $)(r=0.609, p<0.05)$. Also, hepatic insulin resistance showed a significant positive correlation with GOT $(\mathrm{r}=0.467, p<0.05)$, GPT $(\mathrm{r}=0.434$, $p<0.05)$, and $\gamma$-GTP $(\mathrm{r}=0.375, p<0.05)$, which reflect liver functions, and a significant positive correlation with hs-CRP $(\mathrm{r}=0.492, p<0.05)$. Hepatic insulin resistance also showed a significant positive correlation with serum total cholesterol

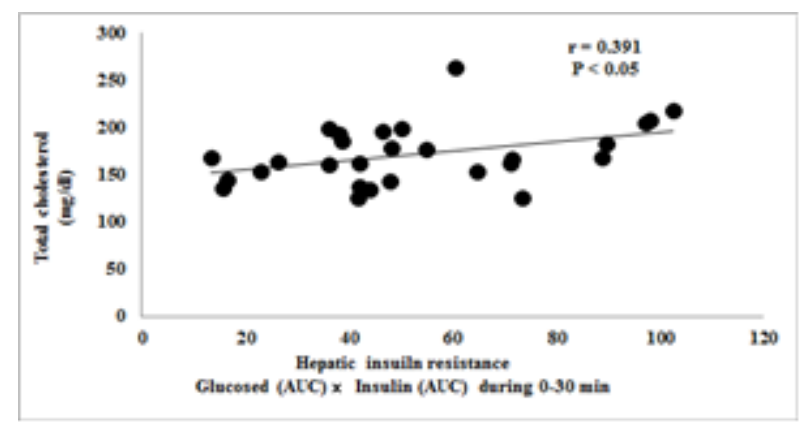

Fig. 1. The correlation between total cholesterol and hepatic insulin resistance level ( $\mathrm{r}=0.391, p<0.05)$, as shown in Fig. 1 . On the other hand, muscle insulin sensitivity showed no correlation with either the body fat or the liver function index $(p>0.05)$. Hepatic insulin resistance showed a significant negative correlation with FFA-IS $(\mathrm{r}=-.386, p<0.05)$ that was calculated from insulin and FFA. For the tissue insulin resistance's correlation with blood components, the liver had more correlation with the liver function, inflammation, and serum lipids than with the muscles.

\section{The correlation with insulin resistance surrogate index}

Variables Table 2 shows a simple correlation with previous insulin surrogate indexes.

For the correlation with the indexes related to insulin sensitivity, muscle insulin sensitivity showed a significant positive correlation with Avignon $(\mathrm{r}=0.493, p<0.05)$ and Matsuda index $(\mathrm{r}=0.577, p<0.05)$ but showed a significant negative correlation with HOMA- $\% \mathrm{~B}(\mathrm{r}=-0.452, p<0.05)$, which reflected pancreatic beta cell function. Glucose infusion rate using the clamp method showed a significant positive correlation with muscle insulin sensitivity $(\mathrm{r}=0.415, p<$ 0.05 ) as shown in Fig. 2. In other words, systemic and peripheral insulin sensitivity showed a correlation with muscle

Table 2. Univariate correlations between novel- and surrogate index

\begin{tabular}{lcccc}
\hline \multirow{2}{*}{\multicolumn{1}{c}{ Variables }} & \multicolumn{2}{c}{$\mathrm{hIR}$} & \multicolumn{2}{c}{$\mathrm{mIS}$} \\
\cline { 2 - 5 } & $\mathrm{r}$ & $\mathrm{P}$ & $\mathrm{r}$ & $\mathrm{P}$ \\
\hline HOMA-IR & 0.277 & 0.277 & -0.230 & 0.259 \\
HOMA- $\beta$ & 0.334 & 0.082 & -0.452 & $0.021^{*}$ \\
QUICKI & -0.277 & 0.154 & 0.343 & 0.086 \\
Adipose tissue IR & 0.453 & $0.016^{*}$ & 0.195 & 0.348 \\
Avignon A & -0.341 & 0.076 & 0.493 & $0.010^{*}$ \\
Matsuda Index & -0.361 & 0.059 & 0.577 & $0.002^{*}$ \\
OGIS & -0.339 & 0.078 & 0.253 & 0.213 \\
\hline
\end{tabular}

Date show correlation coefficient, $* p<0.05$

hIR: hepatic insulin resistance, mIS: muscle insulin sensitivity

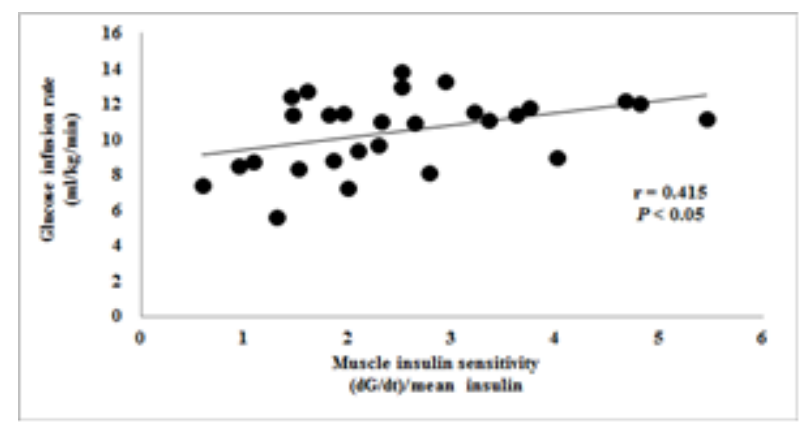

Fig. 2. The correlation between glucose infusion rate and muscle insulin sensitivity 


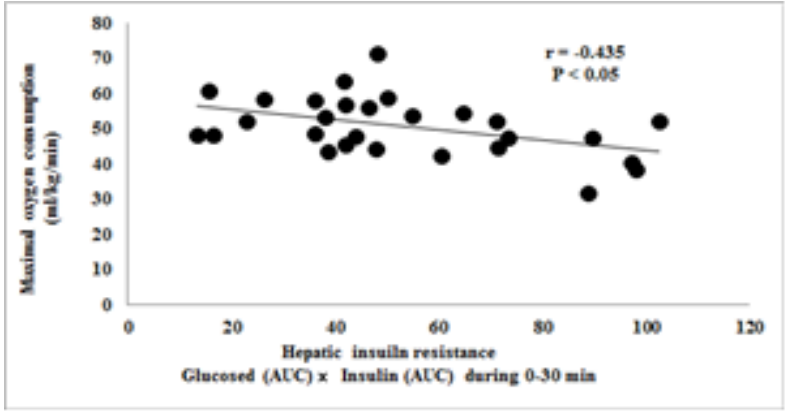

Fig. 3. The correlation between $\mathrm{VO}_{2 \max }$ and hepatic insulin resistance

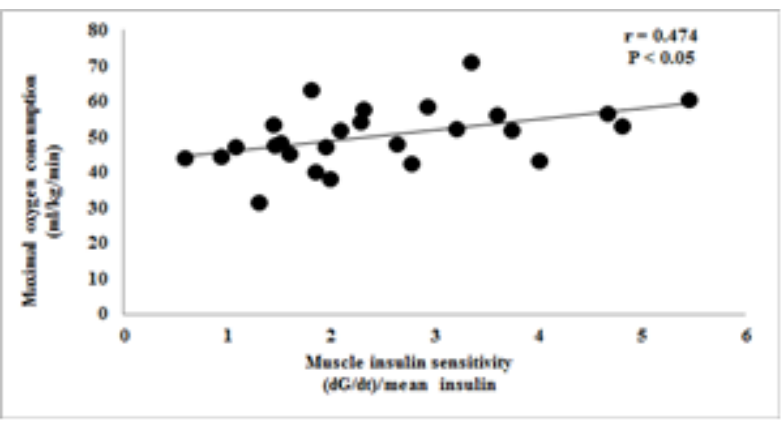

Fig. 4. The correlation between $\mathrm{VO}_{2 \max }$ and muscle insulin sensitivity

insulin sensitivity in young men, but hepatic insulin resistance had no significant correlation with glucose infusion rate $(p$ $>0.05$ ).

The correlation between tissue insulin sensitivity and $\mathrm{VO}_{2 \max }$

There was no problem (data not shown) found from $\mathrm{VO}_{2 \max }$ assessment among all the participants regarding arrhythmia and electrocardiogram during the recovery period. From the correlation with $\mathrm{VO}_{2 \max }$, hepatic insulin resistance showed a significant negative correlation $(\mathrm{r}=-0.435, p<0.05)$, as shown in Fig. 3, while muscle insulin sensitivity showed a significant positive correlation $(\mathrm{r}=0.474, p<0.05)$ as shown in Fig. 4. Therefore, we showed that there was an inverse correlation between tissue insulin resistance and $\mathrm{VO}_{2 \max }$ in young men.

\section{DISCUSSION}

Insulin resistance is related to metabolic syndrome-related diseases, such as hypertension, diabetes, and hyperlipidemia, and this insulin resistance is greatly related to tissues, especially the liver and muscles [5,25]. Although there are various methods to measure systemic insulin resistance, there are many limitations for clinical trials due to the methodological difficulties to measure insulin resistance on local tissues. Despite such difficulties, the surrogate index, which can measure hepatic and muscle insulin resistance from OGTT at the same time, was reported [11].

This study was designed to study the correlation with the surrogate indexes of blood lipids, cardiorespiratory fitness, and insulin resistance to understand the clinical relation among euglycemic hyperinsulinemic clamp method and OGTT-based hepatic insulin resistance in young men. For hepatic insulin resistance, this study found the following results. There was a significant correlation between hepatic insulin resistance and body fat in young men and a significant positive correlation between hepatic insulin resistance and GOT, GPT, and z-GTP, which reflect liver function. Also, hepatic insulin resistance had a significant positive correlation with high-sensitivity C-reactive protein, which was used as an inflammatory marker.

In previous studies, Wallace, et al. reported that, in the group without diabetes, insulin resistance from jugular vein glucose tolerance test was significantly correlated with $\gamma$ -GTP, which was a biomarker of liver cell damage [26]. Also, Jo, et al. reported that, for their study results, GPT and $x-G T P$ could predict metabolic syndrome with insulin resistance of 20,000 or more in number in male and female adults[27].

Hepatic insulin resistance could not be directly measured since it was a large-size epidemiologic study but it suggested that an increase of insulin resistance was related to the increase of hepatic function-related serum enzyme based on the previous studies (of which reported the correlation between hepatic function-related serum enzymes and insulin resistance) and this study's results. From the correlation with insulin resistance surrogate indexes, hepatic insulin resistance showed a significant correlation with FFA-based insulin resistance. Obesity or metabolic syndrome showed high blood FFA and high blood insulin level. The FFA-IS, calculated from the multiplication of fasting FFA and insulin, was related to systemic insulin resistance [24] and showed a significant correlation with hepatic insulin resistance index in this study. As we can see from the statement of Meshkani, et al., hepatic insulin resistance is known to be related to the onset rate of metabolic syndrome and cardiovascular disease [6]. Hepatic insulin resistance from the aspect of blood glucose control was related to fasting hyperglycemia. In other words, inhibiting gluconeogenesis by insulin became impossible and, thus, eventually, hyperglycemia occurred due to the increased release of blood glucose [28].

There was no correlation between fasting blood glucose and hepatic insulin resistance since this study used normal people 
without any disturbed glucose tolerance or glucose metabolism problem but we believed that it was necessary to study blood glucose and hepatic insulin resistance in the groups with diverse ranges of blood glucose in the future since this study used OGTT-based hepatic insulin resistance coming from the multiplication of fasting blood glucose and endogenous gluconeogenesis and the report of significant correlation $(\mathrm{r}=0.64)$ [11].

During the review of clinical characteristics of exercise for hepatic insulin resistance, a significant negative correlation with $\mathrm{VO}_{2 \max }$ was found. The correlation between $\mathrm{VO}_{2 \max }$ and insulin resistance was reported in numerous studies $[12,13]$. However, there is still not enough reports on the correlation between insulin resistance per each tissue and $\mathrm{VO}_{2 \max }$. This study suggested that the people with high maximum aerobic exercise capacity had higher sensitivity in insulin action on the liver. From the context of aerobic exercise training increasing $\mathrm{VO}_{2 \max }$ and systemic insulin sensitivity, additional studies are necessary to investigate the effect of exercise training on hepatic insulin resistance. To be more specific, exercise training increased muscle insulin sensitivity through rapid accumulation of glycogen [29] and Single bout of highintensity aerobic exercise $\left(70 \% \mathrm{VO}_{2 \max }\right)$ increased insulin sensitivity more than moderate-intensity exercise $\left(50 \% \mathrm{VO}_{2 \max }\right)$ [30].

Along with aerobic exercise, resistance exercise also increased insulin sensitivity. Heijden, et al. reported that, using stable isotopes, there was muscle strength and muscle insulin sensitivity increase in obese teenagers aged around 15 who had resistance training which utilized large muscle groups, 2 times per week for 12 weeks, despite of no weight loss [31]. The studies on exercise and insulin sensitivity using conventional methodologies only reported the change of systemic insulin sensitivity based on certain exercise type. Therefore, I believed not only the assessment of systemic insulin resistance using aerobic exercise and resistance exercise but also the direct assessment of the efficacy of exercise, by applying exercise training types (exercise method, intensity, duration, frequency, etc.) on each tissue insulin resistance, is necessary.

The following results were obtained from this study regarding muscle insulin resistance of young, healthy men. First, there was no correlation between muscle insulin sensitivity and body fat or muscle insulin sensitivity and BMI. Therefore, hepatic insulin resistance was more related to body composition distribution within the sample group of young men than muscle insulin resistance. Interestingly, muscle insulin sensitivity had a high correlation with surrogate markers of insulin resistance. For example, it showed a significant positive correlation with Avignon and Matsuda index but showed a significant negative correlation with HOMA- $\% \mathrm{~B}$, which reflected pancreatic beta cell function. Glucose infusion rate derived from euglycemic hyperinsulinemic clamp method, which showed systemic and, especially, peripheral insulin resistance, had a significant positive correlation with muscle insulin sensitivity $(r=0.448)$.

On the contrary, there was no significant correlation between hepatic insulin resistance and glucose infusion rate $(p>0.05)$. As we can see from the correlation among Matsuda index, Avignon, and the clamp method, the blood glucose change (muscle insulin sensitivity) after the maximum blood glucose level caused by OGTT-based insulin control was related to systemic and peripheral insulin sensitivity. From the insulin action on the clinical characteristics of muscle exercise, a significant negative correlation with $\mathrm{VO}_{2 \max }$ was confirmed.

Skeletal muscle insulin resistance was a major characteristic showing in the initial stage of type 2 diabetes and muscle contraction capacity was very important for insulin to bring glucose into muscle cells [7]. This study could not directly investigate the physical activities of the subjects but it appeared to be that, as with the previous study [32] reporting high $\mathrm{VO}_{2 \max }$ in the people who did much physical activities during the day or regular exercise training, the correlation of muscle insulin sensitivity and $\mathrm{VO}_{2 \max }$, which reflected maximum aerobic exercise capacity, has the same meaning as with the aforementioned study in terms of theory.

\section{CONCLUSION}

The following results were obtained after reviewing the clinical characteristics of hepatic and muscle insulin resistance from OGTT in young men who were in their 20's. First, hepatic insulin sensitivity was an indicator reflecting liver function, body fat, and blood total cholesterol and, on the other hand, muscle insulin sensitivity was related to the indicator reflecting peripheral insulin sensitivity. Second, there was a correlation between hepatic and muscle insulin resistance and $\mathrm{VO}_{2 \max }$, meaning that there was a correlation between maximum aerobic exercise capacity and tissue insulin resistance. This method, which was designed to assess hepatic and muscle insulin resistance at the same time using 1-time OGTT, will be used in various studies on the diseases and treatments related to glucose metabolism and, thus, it will be widely utilized in the area of exercise science (physiology and nutrition) in the future. 


\section{REFERENCES}

[1] Gagliardino JJ. Physiological endocrine control of energy homeostasis and postprandial blood glucose levels. Eur Rev Med Pharmacol Sci. 2005;9:75-92.

[2] Frost RJ, Olson EN. Control of glucose homeostasis and insulin sensitivity by the Let-7 family of microRNAs. Proc Natl Acad Sci U S A. 2011;108:21075-21080.

[3] McCarthy MI. Genomics, type 2 diabetes, and obesity. N Engl J Med. 2010;363:2339-2350.

[4] DeFronzo RA, Tobin JD, Andres R. Glucose clamp technique: a method for quantifying insulin secretion and resistance. Am J Physiol. 1979;237:E214-223.

[5] Defronzo RA. Banting Lecture. From the triumvirate to the ominous octet: a new paradigm for the treatment of type 2 diabetes mellitus. Diabetes. 2009;58:773-795.

[6] Meshkani R, Adeli K. Hepatic insulin resistance, metabolic syndrome and cardiovascular disease. Clin Biochem. 2009;42:1331-1346.

[7] DeFronzo RA, Tripathy D. Skeletal muscle insulin resistance is the primary defect in type 2 diabetes. Diabetes Care. 2009;2:S157-163.

[8] Kato K, Takamura T, Takeshita Y, Ryu Y, Misu H, Ota T, Tokuyama K, Nagasaka S, Matsuhisa M, Matsui O, Kaneko S. Ectopic fat accumulation and distant organspecific insulin resistance in Japanese people with nonalcoholic fatty liver disease. PLoS One. 2014;9:e92170.

[9] Bergman RN, Ider YZ, Bowden CR, Cobelli C. Quantitative estimation of insulin sensitivity. Am J Physiol. 1979;236:E667-677.

[10] Ji-Hoon Kim, Lee-Young Lee and Si-Hoon Lee. The assessment and clinical application of insulin resistance. Endocrinology and Metabolism. 2009;24:75-83.

[11] Abdul-Ghani MA, Matsuda M, Balas B, DeFronzo RA. Muscle and liver insulin resistance indexes derived from the oral glucose tolerance test. Diabetes Care. 2007;30:89-94.

[12] Ouyang P, Sung J, Kelemen MD, Hees PS, DeRegis JR, Turner KL, Bacher AC, Stewart KJ. Relationships of insulin sensitivity with fatness and fitness and in older men and women. J Womens Health (Larchmt). 2004;13: 177-185.

[13] Messier V, Malita FM, Rabasa-Lhoret R, Brochu M, Karelis AD. (2008) Association of cardiorespiratory fitness with insulin sensitivity in overweight and obese postmenopausal women: a Montreal Ottawa New Emerging Team study. Metabolism. 2008;57:1293-1298.

[14] Leite SA, Monk AM, Upham PA, Bergenstal RM. Low cardiorespiratory fitness in people at risk for type 2 diabetes: early marker for insulin resistance. Diabetol Metab Syndr. 2009;1:8.

[15] Larsen FJ, Anderson M, Ekblom B, Nystrom T. Cardiorespiratory fitness predicts insulin action and secretion in healthy individuals. Metabolism. 2012;61:12-16.

[16] Kim MK, Tomita, T, Kim MJ, Sasai H, Maeda S, Tanaka K. Aerobic exercise training reduces epicardial fat in obese men. J Appl Physiol. 2009;106:5-11.

[17] Tamura Y, Watada H, Sato F, Kumashiro N, Sakurai Y, Hirose T, Tanaka Y, Kawamori R. Effects of metformin on peripheral insulin sensitivity and intracellular lipid contents in muscle and liver of overweight Japanese subjects. Diabetes Obes Metab. 2008;10:733-738.

[18] Mari A, Pacini G, Murphy E, Ludvik B, Nolan JJ. A model-based method for assessing insulin sensitivity from the oral glucose tolerance test. Diabetes Care. 2001;24:539-548.

[19] Matthews DR, Hosker JP, Rudenski AS, Naylor BA, Treacher DF, Turner RC. Homeostasis model assessment: insulin resistance and beta-cell function from fasting plasma glucose and insulin concentrations in man. Diabetologia. 1985;28:412-419.

[20] Katz A, Nambi SS, Mather K, Baron AD, Follmann DA, Sullivan G, Quon MJ. Quantitative insulin sensitivity check index: a simple, accurate method for assessing insulin sensitivity in humans. J Clin Endocrinol Metab. 2000;85:2402-2410.

[21] Wallace TM, Levy JC, Matthews DR. Use and abuse of HOMA modeling. Diabetes Care. 2004;27:1487-1495.

[22] Matsuda M, DeFronzo RA. Insulin sensitivity indices obtained from oral glucose tolerance testing: comparison with the euglycemic insulin clamp. Diabetes Care. 1999;22;1462-1470.

[23] Avignon A, Boegner C, Mariano-Goulart D, Colette C, Monnier L. Assessment of insulin sensitivity from plasma insulin and glucose in the fasting or post oral glucoseload state. Int J Obes Relat Metab Disord. 1999;23: 512-517.

[24] Belfiore F, Iannello S, Volpicelli G. Insulin sensitivity indices calculated from basal and OGTT-induced insulin, glucose, and FFA levels. Mol Genet Metab. 1998;63: 134-141.

[25] Maeng-Kyu Kim. Ectopic fat tissue and metabolism. The Korean Journal of Obesity. 2011;20:99-106.

[26] Wallace TM, Utzschneider KM, Tong J, Carr DB, Zraika S, Bankson DD, Knopp RH, Kahn SE. Relationship of liver enzymes to insulin sensitivity and intra-abdominal fat. Diabetes Care. 2007;30:2673-2678.

[27] Jo SK, Lee WY, Rhee EJ, Won JC, Jung CH, Park CY, 
Oh KW, Park SW, Kim SW. Serum gamma-glutamyl transferase activity predicts future development of metabolic syndrome defined by 2 different criteria. Clin Chim Acta. 2009;403:234-240.

[28] Bock G, Chittilapilly E, Basu R, Toffolo G, Cobelli C, Chandramouli V, Landau BR, Rizza RA. Contribution of hepatic and extrahepatic insulin resistance to the pathogenesis of impaired fasting glucose: role of increased rates of gluconeogenesis. Diabetes. 2007;56:1703-11.

[29] Holloszy JO. Exercise-induced increase in muscle insulin sensitivity. J Appl Physiol. 2005;99:338-343.

[30] Hayashi Y, Nagasaka S, Takahashi N, Kusaka I, Ishibashi S, Numao S, Lee DJ, Taki Y, Ogata H, Tokuyama K,
Tanaka K. A single bout of exercise at higher intensity enhances glucose effectiveness in sedentary men. J Clin Endocrinol Metab. 2005;90:4035-4040.

[31] Gert-Jan van der Heijden, Zhiyue J. Wang, Zili Chu, Gianna Toffolo, Erica Manesso, Pieter J.J. Sauer, Agneta L. Sunehag. Strength Exercise Improves Muscle Mass and Hepatic Insulin Sensitivity in Obese Youth. Med Sci Sports Exerc. 2010;42:1973-1980.

[32] Armstrong N, Tomkinson G, Ekelund U. Aerobic fitness and its relationship to sport, exercise training and habitual physical activity during youth. Br J Sports Med. 2011; 45:849-858. 